where $p, n, d \equiv 0(\bmod 1) ; n<p ; c=0,1,2, \cdots, d-1$; and $m, q=0$, $\pm 1, \pm 2, \cdots$.

Thus we have obtained transformations of the Mirimanoff and Vandiver congruences connected with the solution of equation (1.1). Other, and in some cases more symmetric, transformations of these congruences are possible by using one of the other permissible forms for the quadratic functional equation (2.11).

Purdue University

\title{
DEVELOPMENT OF CERTAIN QUADRATIC FUNCTIONAL EQUATIONS
}

HAROLD F. S. JONAH

1. Introduction. In some work devoted to the derivation of certain congruences connected with the solution of Fermat's Last Theorem, it was found necessary to develop several quadratic functional equations of a particular function which we shall define later. This note will deal with the derivation of these functional equations. Maier ${ }^{1}$ derived two such quadratic functional equations for a generating function of the Bernoulli polynomials. This work of Maier serves as the basis for our developments.

2. The Maier results. The function, $f(x, u)$, used by Maier was defined by the infinite series

$$
\sum_{r=-\infty}^{+\infty} \frac{e^{2 \pi i x r}}{u+r}
$$

where $x$ is a real variable satisfying the inequality $0<x<1$, and where $u$ is a real variable subject to the restriction $u \neq 0(\bmod 1)$. Maier, then, showed that if $u, v, x, \xi$ are such that $u, v,(u+v) \neq 0(\bmod 1)$ and $0<\xi<x<1$, that the function $f(x, u)$ is a solution of the functional equation

$$
\begin{aligned}
f(x, u) f(\xi, v)= & f(\xi, u+v) f(x-\xi, u) \\
& -f(x, u+v) f(x-\xi,-v) .
\end{aligned}
$$

Received by the editors April 21, 1944.

${ }^{1}$ W. Maier, Zur Theorie der elliptischen Funktionen, Math. Ann. vol. 104 (1930) pp. 745-769. 
If the restriction on $x$ and $\xi$ is replaced by the condition $0<\xi<1-x$ $<1$ (since we must have $0<x+\xi<1$ ), Maier obtains the functional equation

$$
\begin{aligned}
f(x, u) f(\xi, v)= & f(x, u-v) f(x+\xi, v) \\
& +f(\xi, v-u) f(x+\xi, u) .
\end{aligned}
$$

Returning to the definition of the function $f(x, u),(2.1)$, we note that by considering a special contour integral it has been shown ${ }^{2}$ that

$$
f(x, u)=2 \pi i e^{-2 \pi i x u} /\left(1-e^{-2 \pi i u}\right), \quad u \neq 0(\bmod 1) .
$$

In this form the function $f(x, u)$ is defined for all values of $x$, and we shall now use this form of the function $f(x, u)$. Further we note that the addition-theorems (2.2) and (2.3) must remain satisfied by the function $f(x, u)$ when the form (2.4) is used.

3. The function $f_{p}(x, u)$. For our purposes it was necessary to introduce a new function, which we denote by $f_{p}(x, u)$ and which will be defined in terms of values of the function $f(x, u)$. We define

$$
f_{p}(x, u)=f(x+p, u)-f(x, u), \quad p \equiv 0(\bmod 1) .
$$

Hence by means of $(2.4)$, we have

$$
f_{p}(x, u)=\left[e^{-2 \pi i p u}-1\right] f(x, u) .
$$

Thus

$$
f(x, u)=f_{p}(x, u) /\left(e^{-2 \pi i p u}-1\right) .
$$

By means of (3.2) we can show that

$$
f_{p}(x, u)+f_{-p}(1-x,-u) \equiv 0 .
$$

Other formulae involving the function $f_{p}(x, u)$ which shall prove to be useful for our immediate purpose are

$$
\begin{aligned}
f_{p}(x+p, u) & =e^{-2 \pi i p u} f_{p}(x, u), \\
f_{-p}(x, u) & =-f_{p}(x-p, u)=e^{2 \pi i p u} f_{p}(x, u) .
\end{aligned}
$$

In the above formulae for $f_{p}(x, u)$ we can replace $u$ by $u+\alpha$, where the condition $u \neq 0(\bmod 1)$ now becomes $(u+\alpha) \neq \equiv 0(\bmod 1)$, and everything else remains valid.

${ }^{2}$ Namely, the integral $I_{n}=\int C^{n}\left(e^{(2 \mu-1) \varepsilon \pi i} / \sin \pi z\right) \cdot(d z /(z+u))$, where $0<\mu<1$, $u \neq 0(\bmod 1)$ and where $C_{n}$ is a rectangle with the sides $x= \pm(2 n+1 / 2)$ and $y= \pm m$ with $m$ an arbitrarily large positive number and $n$ as an arbitrarily large positive integer so chosen that $z=-u$ lies in the interior of the rectangle $C_{n}$. Finally it has been shown that $\lim _{m, n \rightarrow \infty} I_{n}=0$. 
4. Addition-theorems for the function $f_{p}(x, u)$. In $\$ 2$ we gave the two addition-theorems for the function $f(x, u)$. In this paragraph we shall derive similar addition-theorems for the function $f_{p}(x, u)$.

Let us write the addition-theorem (2.2) in the form

$$
\begin{aligned}
f(x, u+\alpha) f(\xi, v+\beta)= & f(\xi, u+v+\alpha+\beta) f(x-\xi, u+\alpha) \\
& -f(x, u+v+\alpha+\beta) f(x-\xi,-v-\beta),
\end{aligned}
$$

where $(u+\alpha),(v+\beta),(u+v+\alpha+\beta) \not \equiv 0(\bmod 1)$.

On applying (3.3) to the equation (4.1) we have

This reduces to

$$
\begin{aligned}
& \frac{f_{p}(x, u+\alpha) f_{p}(\xi, v+\beta)}{\left[e^{-2 \pi i p(u+\alpha)}-1\right]\left[e^{-2 \pi i p(v+\beta)}-1\right]} \\
& =\frac{f_{p}(\xi, u+v+\alpha+\beta) f_{p}(x-\xi, u+\alpha)}{\left[e^{-2 \pi i p(u+v+\alpha+\beta)}-1\right]\left[e^{-2 \pi i p(u+\alpha)}-1\right]} \\
& \quad-\frac{f_{p}(x, u+v+\alpha+\beta) f_{p}(x-\xi,-v-\beta)}{\left[e^{-2 \pi i p(u+v+\alpha+\beta)}-1\right]\left[e^{2 \pi i p(v+\beta)}-1\right]} .
\end{aligned}
$$

$$
\begin{gathered}
{\left[e^{-2 \pi i p(u+v+\alpha+\beta)}-1\right] f_{p}(x, u+\alpha) f_{p}(\xi, v+\beta)} \\
=\left[e^{-2 \pi i p(v+\beta)}-1\right] f_{p}(\xi, u+v+\alpha+\beta) f_{p}(x-\xi, u+\alpha) \\
-\left[e^{-2 \pi i p(u+\alpha)}-1\right] \frac{\left[e^{-2 \pi i p(v+\beta)}-1\right]}{\left[e^{2 \pi i p(v+\beta)}-1\right]} \\
\cdot f_{p}(x, u+v+\alpha+\beta) f_{p}(x-\xi,-v-\beta) .
\end{gathered}
$$

In order to further simplify the above equation, we write $\left[e^{-2 x i p(v+\beta)}\right.$ $-1]=\left[e^{-2 \pi i p(u+v+\alpha+\beta)} e^{2 \pi i p(u+\alpha)}-1\right]$, and similarly

$$
\begin{aligned}
& {\left[e^{-2 \pi i p(u+\alpha)}-1\right] \frac{\left[e^{-2 \pi i p(v+\beta)}-1\right]}{\left[e^{2 \pi i p(v+\beta)}-1\right]}} \\
& \quad=\left[e^{-2 \pi i p(u+\alpha)}-1\right] \frac{\left[e^{-2 \pi i p(v+\beta)}-1\right]}{\left[1-e^{-2 \pi i p(v+\beta)}\right]} e^{-2 \pi i p(v+\beta)} \\
& \quad=-\left[e^{-2 \pi i p(u+\alpha)}-1\right] e^{-2 \pi i p(v+\beta)}=-\left[e^{-2 \pi i p(u+v+\alpha+\beta)}-e^{-2 \pi i p(v+\beta)}\right] .
\end{aligned}
$$

Then (4.3) reduces to

$$
\begin{aligned}
{\left[e^{-2 \pi i p(u+v+\alpha+\beta)}-1\right] } & f_{p}(x, u+\alpha) f_{p}(\xi, v+\beta) \\
= & {\left[e^{-2 \pi i p(u+v+\alpha+\beta)} e^{2 \pi i p(v+\beta)}-1\right] } \\
& \cdot f_{p}(\xi, u+v+\alpha+\beta) f_{p}(x-\xi, u+\alpha) \\
& +\left[e^{-2 \pi i p(u+v+\alpha+\beta)}-e^{-2 \pi i p(v+\beta)}\right] \\
& \times f_{p}(x, u+v+\alpha+\beta) f_{p}(x-\xi,-v-\beta) .
\end{aligned}
$$


Then on making use of (3.5) we obtain a quadratic functional equation of the function $f_{p}(x, u)$, namely

$$
\begin{aligned}
f_{p}(x+p, u+ & \alpha) f_{p}(\xi+p, v+\beta)-f_{p}(x, u+\alpha) f_{p}(\xi, v+\beta) \\
= & f_{p}(\xi+p, u+v+\alpha+\beta) f_{p}(x-\xi-p, u+\alpha) \\
& -f_{p}(\xi, u+v+\alpha+\beta) f_{p}(x-\xi, u+\alpha) \\
& +f_{p}(x+p, u+v+\alpha+\beta) f_{p}(x-\xi,-v-\beta) \\
& -f_{p}(x, u+v+\alpha+\beta) f_{p}(x-\xi-p,-v-\beta),
\end{aligned}
$$

where $(u+\alpha),(v+\beta),(u+v+\alpha+\beta) \not \equiv 0(\bmod 1), p \equiv 0(\bmod 1)$.

Next, on using (3.4) with (4.5) we obtain for the latter equation the following modified form:

$$
\begin{aligned}
- & f_{-p}(1-x-p,-u-\alpha) f_{p}(\xi+p, v+\beta) \\
& +f_{-p}(1-x,-u-\alpha) f_{p}(\xi, v+\beta) \\
= & -f_{p}(\xi+p, u+v+\alpha+\beta) f_{-p}(1-x+\xi+p,-u-\alpha) \\
& +f_{p}(\xi, u+v+\alpha+\beta) f_{-p}(1-x+\xi,-u-\alpha) \\
& +f_{-p}(1-x-p,-u-v-\alpha-\beta) f_{-p}(1-x+\xi, v+\beta) \\
& -f_{-p}(1-x,-u-v-\alpha-\beta) f_{-p}(1-x+\xi+p, v+\beta) .
\end{aligned}
$$

Consequently, on replacing $1-x$ by $x$ and $-u-\alpha$ by $u+\alpha,(4.6)$ becomes

$$
\begin{aligned}
f_{-p}(x-p, u+ & \alpha) f_{p}(\xi+p, v+\beta)-f_{-p}(x, u+\alpha) f_{p}(\xi, v+\beta) \\
= & f_{p}(\xi+p, v-u+\beta-\alpha) f_{-p}(x+\xi+p, u+\alpha) \\
& -f_{p}(\xi, v-u+\beta-\alpha) f_{-p}(x+\xi, u+\alpha) \\
& +f_{-p}(x, u-v+\alpha-\beta) f_{-p}(x+\xi+p, v+\beta) \\
& -f_{-p}(x-p, u-v+\alpha-\beta) f_{-p}(x+\xi, v+\beta) .
\end{aligned}
$$

Introducing (3.6) in equation (4.7) we obtain the following functional equation for the function $f_{p}(x, u)$,

$$
\begin{aligned}
& f_{p}(x-p, u+\alpha) f_{p}(\xi, v+\beta)-f_{p}(x-2 p, u+\alpha) f_{p}(\xi+p, v+\beta) \\
&= f_{p}(\xi, v-u+\beta-\alpha) f_{p}(x+\xi+p, u+\alpha) \\
&-f_{p}(\xi+p, v-u+\beta-\alpha) f_{p}(x+\xi, u+\alpha) \\
&+f_{p}(x-p, u-v+\alpha-\beta) f_{p}(x+\xi, v+\beta) \\
&-f_{p}(x-2 p, u-v+\alpha-\beta) f_{p}(x+\xi+p, v+\beta),
\end{aligned}
$$

where $(u+\alpha),(v+\beta),(u-v+\alpha-\beta) \not \equiv 0(\bmod 1)$. 
The addition-theorem (2.3) for the function $f(x, u)$ may be written in the form

$$
\begin{aligned}
f(x, u+\alpha) f(\xi, v+\beta)= & f(x, u-v+\alpha-\beta) f(x+\xi, v+\beta) \\
& -f(\xi, v-u+\beta-\alpha) f(x+\xi, u+\alpha),
\end{aligned}
$$

where $(u+\alpha),(v+\beta),(u-v+\alpha-\beta) \not \equiv 0(\bmod 1)$.

Then on applying (3.3) to the equation (4.9) and simplifying, we obtain the following addition-law

$$
\begin{aligned}
f_{p}(x+p, u+ & \alpha) f_{p}(\xi-p, v+\beta)-f_{p}(x, u+\alpha) f_{p}(\xi, v+\beta) \\
= & f_{p}(x+p, u-v+\alpha-\beta) f_{p}(x+\xi+p, v+\beta) \\
& -f_{p}(x, u-v+\alpha-\beta) f_{p}(x+\xi, v+\beta) \\
& +f_{p}(\xi-p, v-u+\beta-\alpha) f_{p}(x+\xi, u+\alpha) \\
& -f_{p}(\xi, v-u+\beta-\alpha) f_{p}(x+\xi+p, u+\alpha),
\end{aligned}
$$

where $p \equiv 0(\bmod 1)$ and $(u+\alpha),(v+\beta),(u-v+\alpha-\beta) \neq 0(\bmod 1)$.

If we apply (3.4) to (4.10), then replace $1-x$ by $x$ and $-u-\alpha$ by $u+\alpha$, and finally apply (3.6), we obtain another functional equation

$$
\begin{aligned}
f_{p}(x-2 p, & u+\alpha) f_{p}(\xi-p, v+\beta)-f_{p}(x-p, u+\alpha) f_{p}(\xi, v+\beta) \\
= & f_{p}(x-2 p, u+v+\alpha+\beta) f_{p}(x-\xi-2 p,-v-\beta) \\
& -f_{p}(x-p, u+v+\alpha+\beta) f_{p}(x-\xi-p,-v-\beta) \\
& +f_{p}(\xi-p, u+v+\alpha+\beta) f_{p}(x-\xi-p, u+\alpha) \\
& -f_{p}(\xi, u+v+\alpha+\beta) f_{p}(x-\xi-2 p, u+\alpha),
\end{aligned}
$$

where $(u+\alpha),(v+\beta),(u+v+\alpha+\beta) \not \equiv 0(\bmod 1)$ and $p \equiv 0(\bmod 1)$.

Thus we have derived four addition-theorems for the function $f_{p}(x, u)$. These functional equations were used in connection with the solution of a certain diophantine equation.

Purdue University 